\title{
Panuveitis and multifocal retinitis in a patient with leucocytoclastic vasculitis
}

\author{
James C Tsai, David J Forster, Richard R Ober, Narsing A Rao
}

To our knowledge, bilateral panuveitis and multifocal retinitis/vasculitis have not been described previously in association with leucocytoclastic vasculitis. We describe a patient with bilateral panuveitis associated with multifocal retinitis/vasculitis who also had multiple purpuric, palpable skin lesions. Biopsies of the skin lesions were diagnostic of leucocytoclastic vasculitis. An extensive examination for systemic disease was otherwise negative. Prompt treatment with systemic corticosteroids resulted in dramatic improvement of both the ocular and the skin lesions.

\section{Case report}

A 57-year-old woman was referred for evaluation of recurrent episodes of bilateral uveitis of 9 years' duration. An extensive laboratory investigation, including purified protein derivative, Toxoplasma titres, erythrocyte sedimentation rate (ESR) HLA B-27, and chest $x$ ray had been negative. On review of systems, the patient gave a history of a few episodes of arthritis affecting primarily the right ankle, as well as a psoriaticlike rash of her lower extremities. This rash reportedly had been investigated without positive results at another academic medical centre.

On examination the patient displayed bilateral panuveitis that affected the left eye more than the right eye. Best corrected visual acuity was 20/60 in the right eye and 20/200 in the left eye. Slitlamp examination revealed rare cells in the right anterior chamber and $2+$ cells and $1+$ flare in the left eye. There were $1+$ vitreous cells in the right eye and $2+$ vitreous cells in the left eye. The right fundus showed slight pallor of the optic disc and some retinal pigment epithelial pigmentary changes inferiorly. The left fundus was remarkable for moderate optic disc oedema and multiple, focal, whitish infiltrates $(200-500 \mu \mathrm{m}$ in size) of the inner retinal layer that were situated throughout the posterior pole. There were also scattered intraretinal haemorrhages. Small areas of preretinal infiltrate were observed along the inferonasal and below the inferotemporal arcades (Fig 1).

Fluorescein angiography demonstrated hypofluorescence associated with the intraretinal haemorrhages in the posterior pole of the left fundus. In the mid phase angiogram, leakage of fluorescein dye from mainly retinal arterioles and capillaries was seen in the parafoveal region of the left eye. Later phases of the angiogram revealed further prominence of this hyperfluorescence, consistent with macular oedema. Hyperfluorescence of the optic nerve head, compatible with disc oedema, was also noted (Fig 2). Fluorescein angiography did not reveal any active vasculitis in the right eye.

A diagnosis of bilateral panuveitis with active multifocal retinitis and vasculitis of the left eye was made. The patient received a depot corticosteroid (40 mg) injection in the left eye, and systemic prednisone therapy, $40 \mathrm{mg}$ a day, was initiated. Follow up examination 2 weeks later revealed improvement of vision in the left eye without evidence of the previously noted inflammatory cells, retinal lesions, or vasculitis. The patient was then tapered off the systemic steroids.

Laboratory investigations included antinuclear antibodies (ANA) titre, rheumatoid factor, syphilis serology, Lyme enzyme-linked immunosorbent assay (ELISA), serum carcinoembryonic antigen (CEA), and angiotensin converting enzyme; results of all of these tests were within normal limits. A systemic collagen vascular disorder such as lupus erythematosus was suspected, but a serum immunology panel for lupus was negative. A computed tomography scan of the brain was also negative. The patient

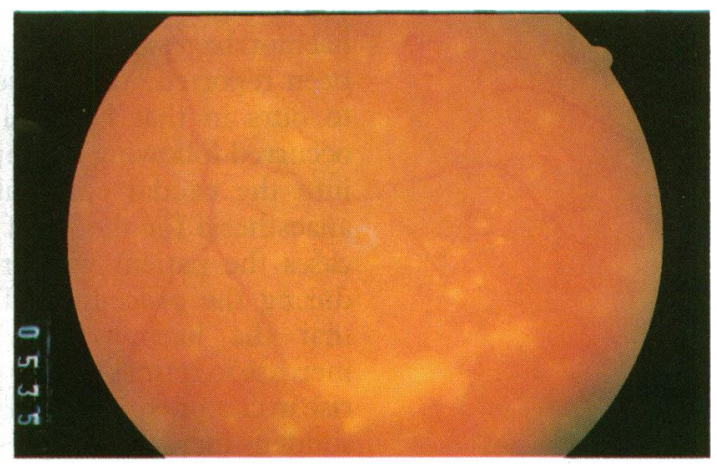

Doheny Eye Institute and the Department of Ophthalmology, University of Southern California School of Medicine, Los Angeles, California, USA JCTsai D J Forster R Obe Correspondence to: Narsing A Rao. MD, Doheny Eye Institute, 1355 San Pablo Street, Los Angeles, CA 90033, USA.

Accepted for publication 12 January 1993
Fig $1 B$

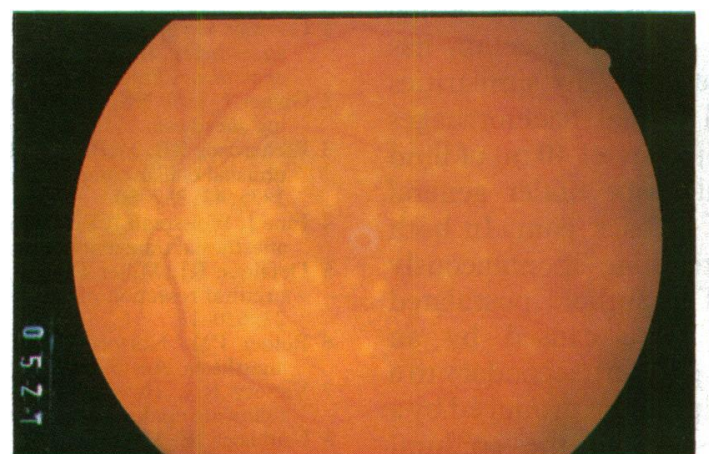

Fig $1 A$

Figure 1 (A) Fundus photograph of left posterior pole. Mild vitreous haze is present, consistent with vitritis. Multiple white intraretinal lesions are scattered throughout the posterior pole, and a few scattered intraretinal haemorrhages are present. Moderate optic disc oedema is noted. (B) Fundus photograph below the left inferotemporal arcade. Small areas of preretinal infiltrates are noted, in addition to the intraretinal lesions. 


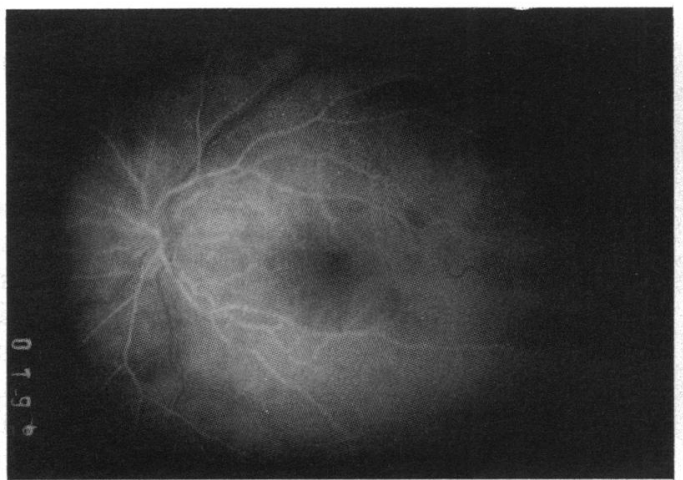

Fig $2 A$

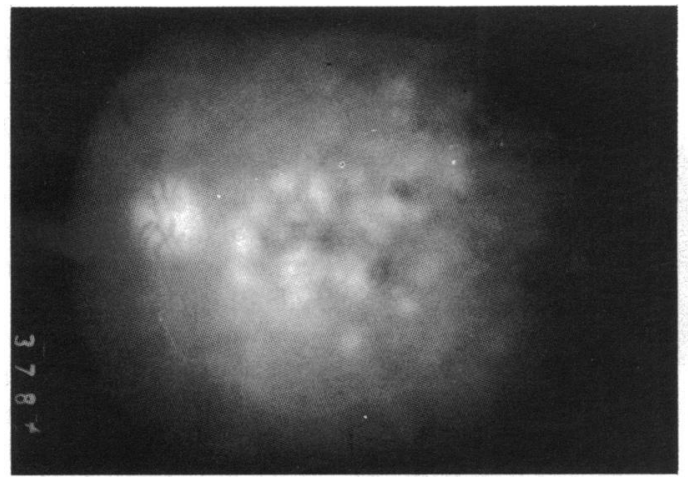

Fig 2C

did have an elevated ESR and 3+ serum C-reactive protein levels.

The patient returned 3 weeks later with an acute 3 day history of rash, headache, fever, and increased floaters in the eyes. A recurrence of the bilateral panuveitis was diagnosed. Examination of the skin showed multiple, purple erythematous lesions, measuring $1-3 \mathrm{~cm}$ in diameter, distributed on the limbs, face, and ears (Fig 3).

Reactivation of the rash prompted a search for the skin biopsies performed previously at another academic medical centre; this search revealed that a previous biopsy of a similar skin lesion of the elbow was positive for leucocytoclastic vasculitis. Upon learning of this diagnosis, we concluded that the episodes of intraocular inflammation were most probably related to this systemic process. Treatment was continued with systemic corticosteroids (prednisone $40 \mathrm{mg}$ a day) as needed to control the skin and ocular manifestations of the disease. Follow up examination 3 weeks later revealed no evidence of intraocular inflammation or of any skin lesions.

\section{Comment}

Leucocytoclastic vasculitis, known also as allergic vasculitis, hypersensitivity vasculitis, or necrotising vasculitis, consists specifically of a vasculitis that affects postcapillary venules of less than $0.1 \mathrm{~mm}$ in diameter.$^{1-3}$ This vasculitic entity is associated most commonly with the connective tissue disease. ${ }^{4}$ Skin lesions are typically ulcerative and purpuric in this entity, with a predilection to occur over the distal extremities." Approximately $50 \%$ of patients with leucocytoclastic vasculitis will have involvement of at least one organ, in addition to the skin. ${ }^{6}$ The

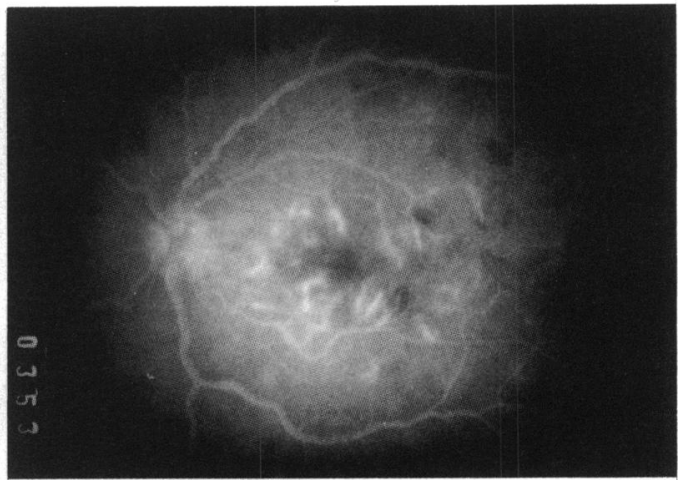

Fig $2 B$

Figure 2 Fluorescein angiogram of left eye. (A)

Arteriovenous phase showing hypofluorescence associated with the scattered intraretinal haemorrhages seen in the colour photographs. (B) Middle phase showing hyperfluorescent staining of vessel walls in posterior pole, characteristic of $a$ vasculitic process. (C) Late phase showing multiple areas of prominent hyperfluorescence in the parafoveal region, consistent with macular oedema. There is also hyperfluorescence of the optic nerve head, compatible with disc oedema.

organs that have been reported to be involved include kidneys, joints, lungs, brain, and gastrointestinal tract ${ }^{7}$; ocular involvement is believed to be rare. The most common laboratory findings include an abnormal ESR, abnormal urinalysis, positive rheumatoid factor, and positive ANA titre. $^{3}$

Ophthalmic manifestations of leucocytoclastic vasculitis are relatively rare, although in the dermatological literature there have been reports suggesting an association of uveitis, episcleritis, pseudotumour cerebri, and optic atrophy in patients with this vasculitis. ${ }^{8}$ Similarly, there are reports of patients with hypocomplementaemic cutaneous vasculitis syndrome (that is, leucocytoclastic vasculitis) who developed bilateral, recurrent iridocyclitis, non-granulomatous anterior uveitis, marginal ulceration of the cornea, inflammatory chemosis, subconjunctival haemorrhage, and possible Posner-Schlossman syndrome. ${ }^{9-11}$

We suspect that in some cases leucocytoclastic vasculitis may indeed be associated with uveitis, skin lesions, and arthritis in patients who have previously had a negative systemic examination. It is known that certain types of endogenous uveitis are immune complex mediated, ${ }^{12}$ so it is quite probable that the circulating immune complexes present in leucocytoclastic vasculitis may incite an endogenous uveitis and associated retinitis. We are aware of a previous report that has implicated an immune complex mediated retinal vasculitis in the setting of polyarteritis nodosa, ${ }^{13} \mathrm{a}$ disease that displays histological features similar to those of leucocytoclastic vasculitis.

The multiple whitish intraretinal infiltrates observed in our patient most probably result from transudation of fluid and white blood cells 


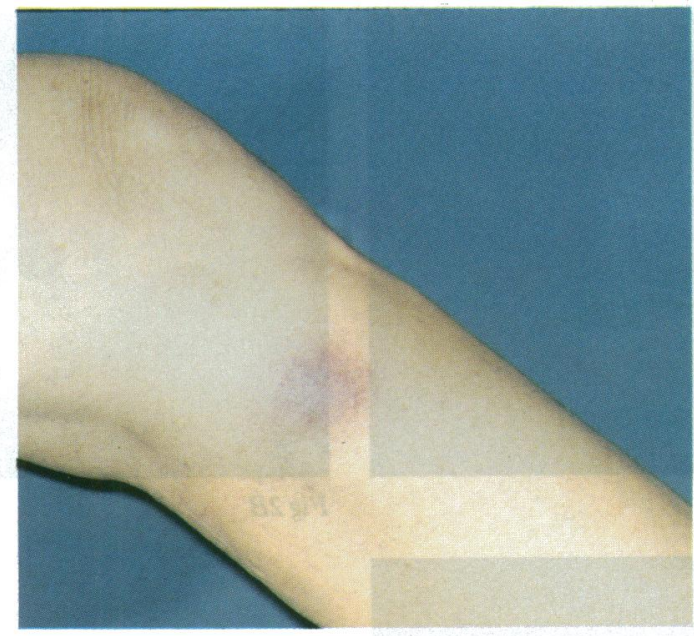

Fig $3 A$

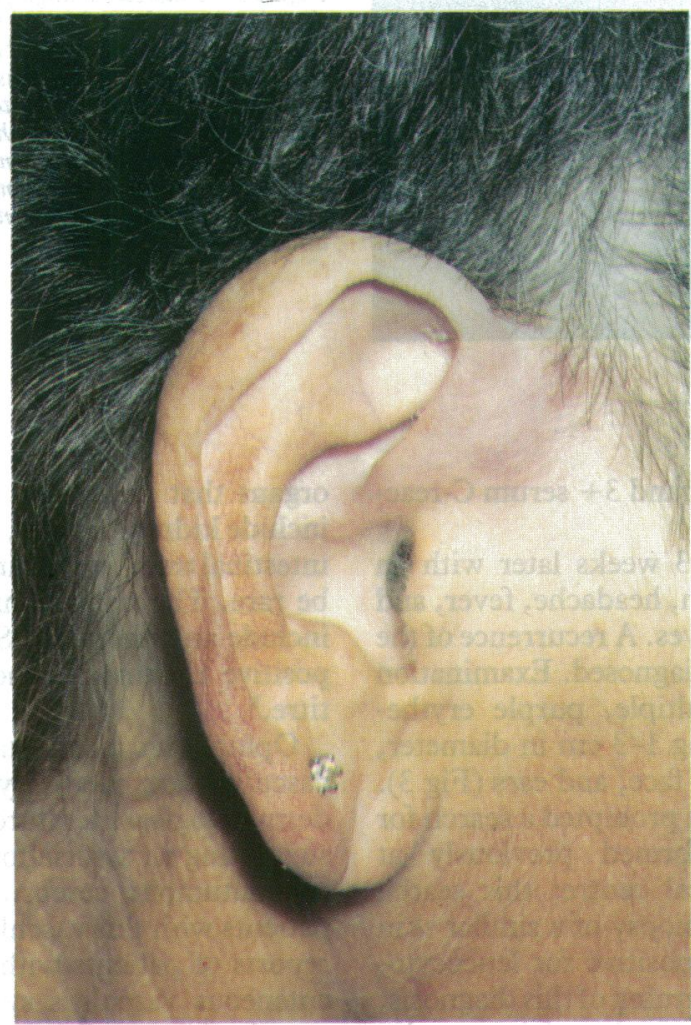

Fig $3 B$

Figure 3 Purpuric, palpable skin lesions. (A) Below the knee. (B) Anterior to the upper aspect of the ear lobe.

from damaged retinal blood vessels. Fluorescein angiography demonstrated a characteristic mid phase leakage from parafoveal vessels. The differential diagnosis of this angiogram pattern includes those systemic vasculitides associated with retinal involvement: systemic lupus erythematosus (SLE), polyarteritis nodosa, sarcoi- dosis, mixed connective tissue disease, dermatomyositis, progressive systemic sclerosis, and Behçet's syndrome. The negative serum immunology panel excluded SLE as a possibility, while the negative angiotensin converting enzyme ruled out sarcoidosis. The clinical systemic features in this case were atypical for dermatomyositis, progressive systemic sclerosis, polyarteritis nodosa, Behçet's syndrome, and mixed connective tissue disease. The histopathological features of the skin biopsy were diagnostic of leucocytoclastic vasculitis.

Treatment of patients with leucocytoclastic vasculitis entails initially the identification or ruling out of any predisposing factors, including connective tissue diseases, infections, drugs, and malignancy. Fluorescein angiography should be performed to rule out any accompanying macular oedema. Our patient did, indeed, have angiographic evidence of macular oedema, although this was not noted on clinical examination. Standard therapy to date consists of systemic corticosteroids initially, with addition of cyclophosphamide if the disease process/ inflammation is unresponsive to steroids. ${ }^{6}$

In the above case, treatment with systemic corticosteroids eliminated the associated retinitis, and we believe that this therapeutic regimen is indicated. Additional agents (that is, cyclophosphamide) may be considered in some particularly recalcitrant cases. The presence of systemic involvement necessitates consultation with an internist/dermatologist in order to optimise the treatment regimen.

1 Fauci AS, Haynes BF, Katz P. The spectrum of vasculitis: clinical, pathologic, immunologic, and therapeutic considerations. Ann Intern Med 1978; 89: 660-76.

2 Sams WM Jr, Thorne EG, Small P, Mass MF, McIntosh RM Stanford RE. Leukocytoclastic vasculitis. Arch Dermato 1976; 112: 219-26.

3 Sanchez NP, Van Hale HM, Su WPD. Clinical and histopathologic spectrum of necrotizing vasculitis: report of findings in 101 cases. Arch Dermatol 1985; 121: 220-4.

4 Gilliam JN, Smiley JD. Cutaneous necrotizing vasculitis and related disorders. Ann Allergy 1976; 37: 328-39.

5 Braverman IM. Skin signs of systemic disease. 2nd ed. Philadelphia: Saunders, 1981: 393-426.

6 Sams WM Jr. Small vessel vasculitis. In: Sams WM Jr, Lynch PJ, eds. Principles and practice of dermatology. New York: Churchill Livingstone, 1990: 523-31

7 Ramsay C, Fry L. Allergic vasculitis: clinical and histological features and incidence of renal involvement. $\mathrm{Br} \mathcal{F}$ Dermatol 1969; 81: 96-102.

8 Sanchez NP, Winkelmann RK,Schroeter AL, Dicken CH The clinical and histopathologic spectrums of urticarial vasculitis: study of forty cases. F Am Acad Dermatol 1982; 7 : va9-605.

9 Corwin JM, Baum J. Iridocyclitis in two patients with hypocomplementemic cutaneous vasculitis. Am $\mathcal{F}$ Ophthalmol 1982; 94: 111-3.

10 Ryan LM, Kozin F, Eiferman R. Immune complex uveitis: a case. Ann Intern Med 1978; 88: 62-3.

11 Doutre MS, Beylot C, Morel P, Dallat A, Lagoutte F, Beylot J, et al. Manifestations ophthalmologiques des vascularites et al. Manifestations ophthalmologiques des vascularites leucocytoclasiques. A propos de
matol Venereol 1986; 113:419-25.

12 Char DH, Stein P, Masi R, Christensen M. Immune complexes in uveitis. Am $\mathcal{F}$ Ophthalmol 1979; 87: 678-81

13 Morgan CM, Foster CS, D'Amico DJ, Gragoudas ES. Retinal vasculitis in polyarteritis nodosa. Retina 1986; 6: 205-9. 